\title{
Effect of Amino and Carboxyl Functionalization on the Photoluminescence Properties of Rice Husk-Derived Carbon Quantum Dots (RH-CQDs)
}

\author{
Khee Chung Hui ${ }^{1}$, Nur Hafizah Zainal Abidin ${ }^{1}$, Nonni Soraya Sambudi1, ${ }^{\text {* }}$ \\ ${ }^{1}$ Department of Chemical Engineering, Universiti Teknologi PETRONAS, Bandar Seri Iskandar, 32610 Perak Darul Ridzuan, Malaysia \\ ${ }^{2}$ Center for Advanced Integrated Membrane System (AIMS), Universiti Teknologi PETRONAS, Bandar Seri Iskandar, 32610 Perak \\ Darul Ridzuan, Malaysia
}

\begin{abstract}
In this work, carbon quantum dots (CQDs) are synthesized using rice husk as a natural precursor. The effect of amino and carboxyl functionalization is studied by adjusting the amount of ethylenediamine (EDA) as the amino source and ascorbic acid as the carboxyl source. HRTEM analysis show the formation of spherical carbon quantum dots. FTIR analysis confirms the presence of $\mathrm{OH}$ and $\mathrm{CO}$ bonding, indicating formation of CQDs. The addition of EDA and ascorbic acid quenches the fluorescence and shifts the emission wavelength from blue region $(450-485 \mathrm{~nm})$ to green region $(500-565 \mathrm{~nm})$. Based on the results, N-RHCQDs $(0.6 \mathrm{ml})$ and C-RHCQDs $(2.5 \mathrm{ml})$ are chosen as the best samples as they give the highest quantum yield of $0.37 \%$ and $3.26 \%$ respectively. This implies that the fluorescence intensity is higher at a more basic and less acidic condition. This study suggests that the addition of different functionalization agents can tune the photoluminescence properties of CQDs that will be beneficial for its application.
\end{abstract}

\section{Introduction}

Carbon quantum dots or carbon dots, as a new class in carbonaceous nanomaterials have received much interest among researchers ever since its first discovery by $\mathrm{Xu}$ et al. (2004) from the purification of carbon nanotubes [1]. Carbon quantum dots are small carbon nanoparticles that exist in quasi spherical form with a size smaller than 10 $\mathrm{mm}$ [2]. The outstanding fluorescence properties, facile synthesis, low cost, chemical inertness, low toxicity, good solubility and good photochemical stability of carbon dots attract interest of researchers to explore their potential technical applications [3]. Recent work on carbon quantum dots have been focused on the utilization of carbon quantum dots in controlled drug delivery, bioimaging, biosensing, photocatalysis, solar cells etc.

Many studies have been done to investigate the synthesis process using different synthetic and natural precursors. Recently, natural precursors are preferable among researchers due to their low-cost and high abundance advantages [4]. There are several reported natural precursors like sugarcane bagasse $[5,6]$, pineapple waste $[7,8]$, bamboo leaf [9], watermelon peel [10], lemon peel [11], pomelo peel [12] and rice husk $[13,14]$. Rice husk is an agricultural waste and a by-product of rice milling processes [15]. In Malaysia, the production of rice is around 1.7 million metric tonnes in the year 2018. The large-scale production of rice can give rise to a huge amount of agro-waste, in which rice husk is one of them. Hence, for this study, rice husk is used as the precursor to synthesize carbon quantum dots due to its abundancy in environment, low cost, facile preparation method when compared to others biowaste precursors. Hydrothermal method, a bottom-up approach is commonly used in synthesizing the carbon quantum dots from natural precursor due to its facile synthesis, low cost and controlled morphology advantages [16].

Carbon quantum dots (CQDs) with tunable photoluminescence are desired due to their demand in sensing, imaging and photocatalysis application. Recently, studies have been focused on the incorporation of functionalization agent onto carbon quantum dots. Functionalization of CQDs is a surface modification process whereby functional groups are introduced onto the surface of CQDs [17]. As mentioned by Yao et al., surface functionalization is an excellent method to tune the photoluminescence and band gap of carbon quantum dots [18]. Functionalization agent helps in the enlargement of band gap between highest occupied molecular orbital (HOMO) and lowest unoccupied molecular orbital (LUMO) for excitation, thus shifting the fluorescence from blue to red emission [19]. In short, tuning photoluminescence by introducing functionalization agent can lead to the distinct properties in carbon quantum dots.

To date, there is limited research in performing a comparative study on the effects of an acid functionalization agent and a basic functionalization agent on the properties of a biowaste-derived carbon

\footnotetext{
* Corresponding author: soraya.sambudi@utp.edu.my
} 
quantum dots. Thus, in this research, carbon quantum dots are synthesized hydrothermally using rice husk as the precursor. Carboxyl functionalization and amino functionalization are studied by adding ascorbic acid and ethylenediamine (EDA) into carbon quantum dots respectively. Subsequently, the effect of the two different functionalization agents on the photoluminescence properties will be further analysed.

\section{Materials and methods}

\subsection{Materials}

Rice husk obtained from Perak Agricultural Department, Malaysia, was used as biowaste precursor for synthesis of carbon dots. Hydrochloric acid, $\mathrm{HCl}$ (37\%, Merck) was used for the treatment of rice husk. Quinine sulphate was obtained from Sigma Aldrich. Ethylenediamine (EDA) (Merck) and ascorbic acid (HmbG Chemicals) were used as functionalizing agent for the rice husk derived carbon quantum dots. Deionized water was used for the solution preparation, dilution and washing purpose. Dialysis bag (MWCO=12000 Da, China OEM) is used for the purification of carbon dots.

\subsection{Hydrothermal synthesis of rice husk-derived carbon quantum dots (RHCQDs)}

Rice husk-derived carbon quantum dots (RH-CQDs) was prepared according to methodology by Wang et al. with some modifications. Firstly, $10 \mathrm{~g}$ of rice husk was washed with DI water. It was then dried and blended into tiny bits. The rice husk was pre-treated with $20 \mathrm{ml} 0.10$ $\mathrm{M} \mathrm{HCl}$, centrifuged at $4000 \mathrm{rpm}$ and rinsed with DI water for three times. The powder was dried at room temperature overnight. To prepare carbon quantum dots, $0.5 \mathrm{~g}$ of dry rice husk powder was added with $50 \mathrm{ml} \mathrm{DI}$ water into Teflon lined autoclave at $190^{\circ} \mathrm{C}$ for 4 hours. After the autoclave was cooled to room, the solution within the autoclave was centrifuged for 15 minutes to remove the excess solid.

\subsection{Hydrothermal synthesis of functionalized RHCQDs}

To prepare amino-functionalized rice husk-derived carbon quantum dots (N-RHCQDs), $0.5 \mathrm{~g}$ treated rice husk powder and $0.2 \mathrm{ml}$ of ethylenediamine (EDA) were added into $50 \mathrm{ml}$ of DI water, sonicated in an ultrasonic bath. The mixture was then be transferred into Teflon lined autoclave and was heated hydrothermally at $190^{\circ} \mathrm{C}$ for 4 hours. After natural cooling to room temperature, the solution was centrifuged for 15 minutes to remove the excess solid. The solution was then collected and dialyzed in a dialysis bag with a cutoff molecular weight of $12000 \mathrm{Da}$ for $24 \mathrm{~h}$. The steps were repeated for $0.4 \mathrm{ml}$ and $0.6 \mathrm{ml}$ ethylenediamine (EDA).

On the other hand, to prepare carboxylfunctionalized rice husk-derived carbon quantum dots (C-RHCQDs), $0.5 \mathrm{~g}$ treated rice husk powder and $2.5 \mathrm{ml}$ of ascorbic acid were added into $50 \mathrm{ml}$ of DI water, sonicated completely in an ultrasonic bath. The mixture was then be transferred into Teflon lined autoclave and was heated hydrothermally at $190^{\circ} \mathrm{C}$ for 4 hours. After natural cooling to room temperature, the solution was centrifuged for 15 minutes to remove the excess solid. The solution was then collected and dialyzed in a dialysis bag with a cutoff molecular weight of $12000 \mathrm{Da}$ for $24 \mathrm{~h}$. The steps were repeated for $5.0 \mathrm{ml}$ and $10.0 \mathrm{ml}$ of ascorbic acid.

\subsection{Characterization and instrumentation}

The morphology and size of liquid RH-CQDs were characterized using Tecnai G2 20 S-Twin HRTEM. The functional groups of RH-CQDs were confirmed using Perkin Elmer IR spectrometer from wavenumber of 500 to $4000 \mathrm{~cm}^{-1}$. The photoluminescence of the samples was verified using Edinburgh Instrument FLS920 photoluminescence spectrophotometer with $350 \mathrm{~nm}$ laser as the excitation source. The UV-Vis analysis was performed by using Shimadzu UV-1800.

\subsection{Quantum yield determination}

Quantum yield (QY) will act as the response (Y) of the experiment. Quantum yield of all the synthesized RHCDs are measured using the formula below:

$$
Q Y=Q_{R}\left(\frac{I}{I_{R}}\right)\left(\frac{A_{R}}{A}\right)\left(\frac{n}{n_{R}}\right)^{2}
$$

where $\mathrm{R}$ is the reference fluorophore (quinine sulphate in $0.1 \mathrm{M}$ sulphuric acid, $\mathrm{Q}_{\mathrm{R}}=0.54$ ), $\mathrm{I}$ is the integral fluorescence peak area, $\mathrm{A}$ is the corresponding absorbance of UV-Vis spectra and $n$ is the refractive index. The ratio $n / n_{R}$ is normally kept to be 1 .

\section{Results and discussions}

\subsection{Morphology of carbon quantum dots}

The morphology of carbon quantum dots was analysed using high resolution transmission electrons microscopy (HRTEM) at a magnification of x690K. HRTEM analysis shows successful formation of carbon quantum dots. Fig. 1. shows that all the CQDs exhibit spherical morphology. All the CQDs exist in amorphous state. The addition of the two functionalization agents (EDA and ascorbic acid) creates clearer spherical dots. For NRHCQDs, the dots are in cluster form with agglomeration. The agglomeration might be due to the high surface energy exist in the CQDs that lead to strong attraction between the nanoparticles [20]. Interestingly, C-RHCQDs show the clearest image of spherical carbon dots among the samples. 

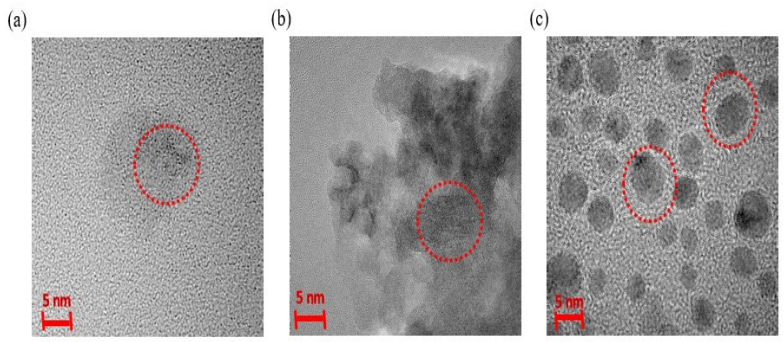

Fig. 1. HRTEM images for (a) RHCQDs, (b) NRHCQDs and (c) C-RHCQDs

\subsection{Functional groups analysis}

FTIR analysis confirms the functional groups present in the carbon quantum dots Fig. 2. illustrates the FTIR spectra of all the synthesized CQDs samples. All the CQDs have the same functional groups being detected. Infrared analysis detects strong absorption at 3331-3340 $\mathrm{cm}-1$, which is due to $\mathrm{O}-\mathrm{H}$ stretching [21]. Apart from that, $\mathrm{C}=\mathrm{O}$ bonding is found at $1636-1638 \mathrm{~cm}-1$ for all synthesized RH-CDs [22]. The amino functional groups that supposedly come from the EDA functionalization are not detected in the spectra owing to the less amount of functionalization being used. For C-RHCQDs, it can be observed that the $\mathrm{C}=\mathrm{O}$ bonding has slightly broader beak when compared to pure RHCQDs, indicating the existence of carboxyl functional groups in the carbon quantum dots.

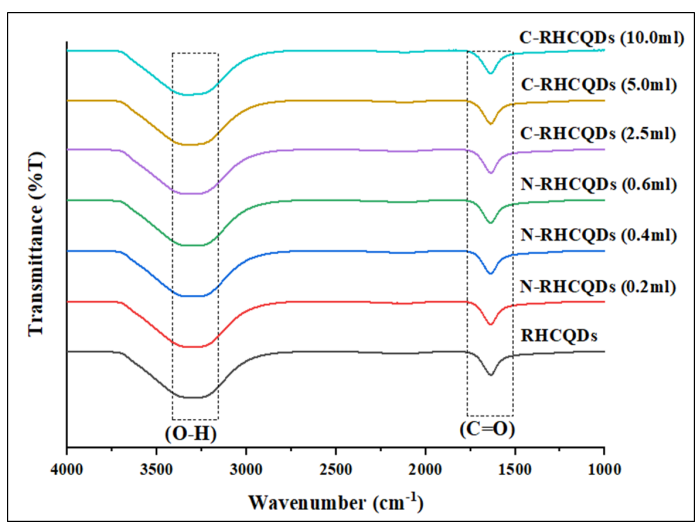

Fig. 2. FTIR spectra of synthesized RHCQDs

\subsection{Optical properties of carbon quantum dots}

Fig. 3. displays the UV-Vis spectra of all the synthesized RHCQDs and functionalized RHCQDs. Table 1 displays the UV-Vis spectroscopic features of all the synthesized CQDs samples. The main absorption peaks for RHCQDs are determined around 212 and $281 \mathrm{~nm}$, which can be ascribed as $n-\pi^{*}$ and $\pi-\pi^{*}$ transitions, respectively. When the functionalization agents are introduced into the CQDs, the UV-Vis curves tend to red shift to a more visible light region. For N-RHCQDs, main absorption peaks are found at $220 \mathrm{~nm}$ and $480 \mathrm{~nm}$, which can be ascribed to $\pi-\pi^{*}$ and $n-\pi^{*}$ transitions respectively. For C-RHCQDs, $\pi-\pi^{*}$ and $n-\pi^{*}$ transitions are determined at $240 \mathrm{~nm}$ and $450 \mathrm{~nm}$ respectively.

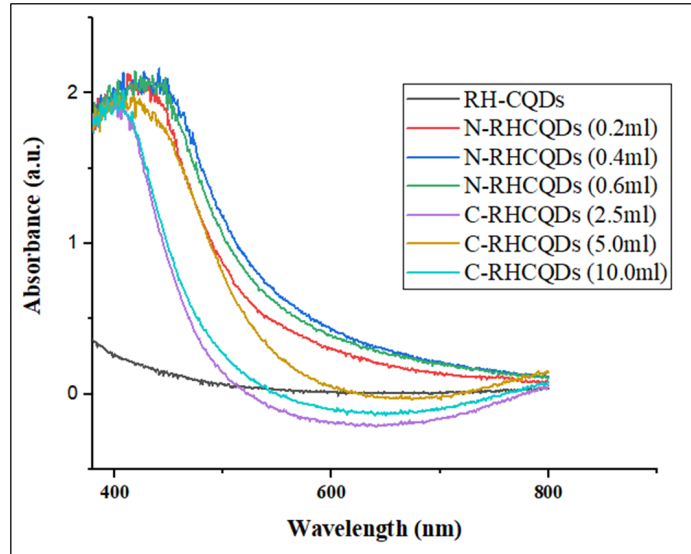

Fig. 3. UV-Vis spectra of all CQDs samples

Table 1: UV-Vis spectroscopic features for all CQDs samples

\begin{tabular}{|l|l|l|}
\hline Sample & Wavelength (nm) & Transition \\
\hline \multirow{2}{*}{ RHCQDs } & 212 & $\mathrm{n} \rightarrow \pi^{*}$ \\
\cline { 2 - 3 } & 281 & $\pi \rightarrow \pi^{*}$ \\
\hline N-RHCQDs & 220 & $\pi \rightarrow \pi^{*}$ \\
\cline { 2 - 3 }$(0.2 \mathrm{ml})$ & 480 & $\mathrm{n} \rightarrow \pi^{*}$ \\
\hline N-RHCQDs & 220 & $\pi \rightarrow \pi^{*}$ \\
\cline { 2 - 3 }$(0.4 \mathrm{ml})$ & 480 & $\mathrm{n} \rightarrow \pi^{*}$ \\
\hline $\begin{array}{l}\text { N-RHCQDs } \\
(0.6 \mathrm{ml})\end{array}$ & 220 & $\pi \rightarrow \pi^{*}$ \\
\cline { 2 - 3 } C-RHCQDs & 480 & $\mathrm{n} \rightarrow \pi^{*}$ \\
$(2.5 \mathrm{ml})$ & 240 & $\pi \rightarrow \pi^{*}$ \\
\hline C-RHCQDs & 450 & $\mathrm{n} \rightarrow \pi^{*}$ \\
$(5.0 \mathrm{ml})$ & 240 & $\pi \rightarrow \pi^{*}$ \\
\hline C-RHCQDs & 450 & $\mathrm{n} \rightarrow \pi^{*}$ \\
$(10.0 \mathrm{ml})$ & 240 & $\pi \rightarrow \pi^{*}$ \\
\cline { 2 - 3 } & 450 & $\mathrm{n} \rightarrow \pi^{*}$ \\
\hline
\end{tabular}

\subsection{Photoluminescence properties of carbon quantum dots}

Fig. 4. shows the graph of photoluminescence (PL) intensity and peak wavelength versus the different amount of functionalization agent. Table 2 shows the calculated quantum yield values for all CQDs samples. From the table and figure below, it can be observed that when EDA is added into the CQDs, the PL intensity and quantum yield decreases. But, when the amount of EDA is varied, the PL intensity and quantum yield show almost similar value due to the little amount being used. However, it is noted that the usage of $0.6 \mathrm{ml}$ EDA gives a slightly higher quantum yield. An increasing amount of basic reagent like will enhance photoluminescence as more negatively charged carbon quantum dots due to deprotonation [23, 24]. In other words, EDA is working as a surface passivating agent in the carbon quantum dots, protecting the surface functional groups on the carbon quantum dots, allowing higher fluorescence intensity [25]. When ascorbic acid is added into CQDs, it also shows lower PL intensity and quantum yield. It gives greater value when more ascorbic acid is added. The drop in the intensity and quantum yield might be due to quenching in fluorescence [26]. Also, when 
functionalization agents are added to the CQDs, the photoluminescence peaks tend to red shift. An increasing amount of acid into carbon quantum dots will lead to protonation of carbon quantum dots, resulting in greater electrostatic force that disrupts the carbon stacking in the dots and leads to lower PL intensity [27, 28].

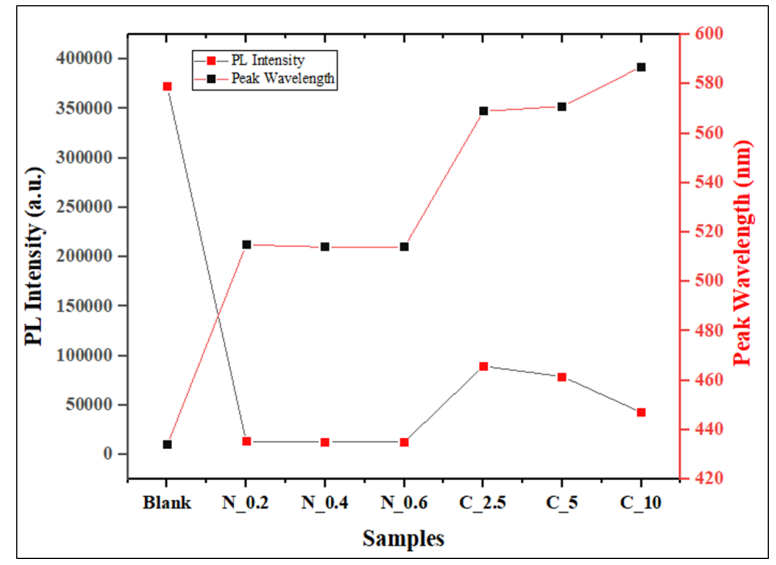

Fig. 4. Fluorescence intensity and peak wavelength of CQDs samples

Table 2: Quantum yield of all CQDs samples

\begin{tabular}{|l|l|}
\hline Sample & Quantum Yield (\%) \\
\hline RHCQDs & 25.80 \\
\hline N-RHCQDs $(0.2 \mathrm{ml})$ & 0.36 \\
\hline N-RHCQDs $(0.4 \mathrm{ml})$ & 0.35 \\
\hline N-RHCQDs $(0.6 \mathrm{ml})$ & 0.37 \\
\hline C-RHCQDs $(2.5 \mathrm{ml})$ & 3.26 \\
\hline C-RHCQDs $(5.0 \mathrm{ml})$ & 2.86 \\
\hline C-RHCQDs $(10.0 \mathrm{ml})$ & 1.24 \\
\hline
\end{tabular}

\section{Conclusion}

This study reports that the variation in amount of functionalization agents can tune the photoluminescence properties of rice husk-derived carbon quantum dots. The addition of EDA and ascorbic acid in carbon quantum dots will shift the emission wavelength from blue to green region. The quenching of fluorescence due to the addition of EDA and ascorbic acid is also observed. Based on the photoluminescence spectroscopy, it can be observed that N-RHCQDs (0.6 $\mathrm{ml})$ and C-RHCQDs $(2.5 \mathrm{ml})$ give the highest fluorescence intensity and quantum yield respectively. It can be concluded that the fluorescence behaviour of CQDs favours more basic and less acidic conditions. Hence, it is proven that photoluminescence of CQDs is tunable under different amount of functionalization agent added during the synthesis.

\section{Acknowledgment}

The author would like acknowledge the funding from Fundamental Research Grant Scheme (FRGS) project with code FRGS/1/2018/TK02/UTP/03/3.

\section{References}

1. X. Xu, R. Ray, Y. Gu, H. Ploehn, L. Gearheart, K. Raker,W. Scrivens, J. Am. Chem. Soc. 126 12736-12737 (2004)

2. R. Wang, K.-Q. Lu, Z.-R. Tang,Y.-J. Xu, J. Mater. Chem. A 5 3717-3734 (2017)

3. K.-W. Chu, L. S. Lee, C.-J. Chang,L. Liu, Polym. J. 11 (2019)

4. V. Sharma, P. Tiwari,M. Shaikh, J. Mater. Chem. B 5 (2017)

5. H. K. Chung, V. Wongso, N. S. Sambudi,Isnaeni, J. Sol-Gel Sci. Technol 93 214-223 (2020)

6. T. S,R. S. D, Appl. Surf. Sci. 390 435-443 (2016)

7. S. A. A. Vandarkuzhali, S. Natarajan, S. Jeyabalan, G. Sivaraman, S. Singaravadivel, S. Muthusubramanian,B. Viswanathan, ACS Omega 3 12584-12592 (2018)

8. P. A. de Yro, G. Quaichon, R. Cruz, C. Emolaga, M. C. Que, E. Magdaluyo,B. Basilia, AIP Conf. Proc. 2083020007 (2019)

9. M. Z. Fahmi, A. Haris, A. J. Permana, D. L. Nor Wibowo, B. Purwanto, Y. L. Nikmah,A. Idris, RSC Adv. 8 38376-38383 (2018)

10. J. Zhou, Z. Sheng, H. Han, M. Zou,C. Li, Mater. Lett. 66 222-224 (2012)

11. A. Tyagi, K. Tripathi, N. Singh, S. Choudhary,R. Gupta, RSC Adv. 6 (2016)

12. W. Lu, X. Qin, S. Liu, G. Chang, Y. Zhang, Y. Luo, A. M. Asiri, A. O. Al-Youbi,X. Sun, Anal. Chem. 84 5351-5357 (2012)

13. V. Wongso, N. Sambudi, S. Sufian,Isnaeni, Biomass Convers. Biorefin. (2020)

14. Z. Wang, J. Liu, W. Wang, Z. Wei, F. Wang, P. Gong, J. Wang, N. Li, B. Liu, Z. Zhang, W. Wang,L. Sun, J. Mater. Chem. B 5 4679-4689 (2017)

15. M. Hema,S. Arivoli, J. Appl. Sci. Environ. Manage. 12 (2010)

16. S. Novais, P. Silva, Z. Macedo,L. Barbosa, Adv. Condens. Matter Phys. 2016 1-7 (2016)

17. B. B. Chen, M. L. Liu, C. M. Li,C. Z. Huang, Adv. Colloid Interface Sci 270 165-190 (2019)

18. B. Yao, H. Huang, Y. Liu,Z. Kang, Trends Chem. 1 (2019)

19. H. Tetsuka, A. Nagoya, T. Fukusumi,T. Matsui, Adv. Mater. 28 n/a-n/a (2016)

20. M. A. Ashraf, W. Peng, Y. Zare,K. Y. Rhee, Nanoscale Res. Lett 13214 (2018)

21. N. Massad-Ivanir, S. Bhunia, N. Raz, E. Segal,R. Jelinek, NPG Asia Mater. 10 e463 (2018)

22. V. Schiopu-Tucureanu, M. Alina,A. Avram, Crit. Rev. Anal. Chem. 46 (2016)

23. S. Dutta Choudhury, J. M. Chethodil, P. M. Gharat, P. P. K,H. Pal, J. Phys. Chem. Lett. 8 1389-1395 (2017) 24. Q. Niu, K. Gao, Z. Lin,W. Wu, Anal. Methods. 5 6228-6233 (2013)

25. K. Dimos, Polym. J. 101312 (2018)

26. F. Zu, F.-y. Yan, Z. Bai, J. Xu, Y. Wang, Y. Huang,X. Zhou, Microchim. Acta 184 1-16 (2017)

27. L. Xu, H. Fan, L. Huang, J. Xia, J. Huang, M. Li, H. Ding, K. Huang,S. Li, J Taiwan Inst Chem Eng 78 247-253 (2017) 
28. M. Zulfajri, S. Dayalan, W.-Y. Li, C.-J. Chang, Y.-

P. Chang,G. G. Huang, Sensors (Basel) 195008 (2019) 